\title{
Synthetic Spectra and Light Curves of Interacting Binaries and Exoplanets with Circumstellar Material: SHELLSPEC
}

\author{
Ján Budaj \\ Astronomical Institute, Tatranská Lomnica, Slovakia, email: budaj@ta3.sk
}

\begin{abstract}
Program SHELLSPEC is designed to calculate light-curves, spectra and images of interacting binaries and extrasolar planets immersed in a moving circumstellar environment which is optically thin. It solves simple radiative transfer along the line of sight in moving media. The assumptions include LTE and optional known state quantities and velocity fields in 3D. Optional (non)transparent objects such as a spot, disc, stream, jet, shell or stars may be defined (embedded) in 3D and their composite synthetic spectrum calculated. The Roche model can be used as a boundary condition for the radiative transfer. Recently, a new model of the reflection effect, dust and Mie scattering were incorporated into the code.

$\epsilon$ Aurigae is one of the most mysterious objects on the sky. Prior modeling of its light-curve assumed a dark, inclined, disk of dust with a central hole to explain the light-curve with a sharp mid-eclipse brightening. Our model consists of two geometrically thick flared disks: an internal optically thick disk and an external optically thin disk which absorbs and scatters radiation. Shallow mid-eclipse brightening may result from eclipses by nearly edge-on flared (dusty or gaseous) disks. Mid-eclipse brightening may also be due to strong forward scattering and optical properties of the dust which can have an important effect on the light-curves.

There are many similarities between interacting binary stars and transiting extrasolar planets. The reflection effect which is briefly reviewed is one of them. The exact Roche shape and temperature distributions over the surface of all currently known transiting extrasolar planets have been determined. In some cases (HAT-P-32b, WASP-12b, WASP-19b), departures from the spherical shape can reach $7-15 \%$.
\end{abstract}

Keywords. binaries: eclipsing, planets and satellites: general

\section{Introduction}

There are sophisticated computer codes for calculating and inverting light curves or spectra of binary stars with various shapes or geometry including the Roche model (Lucy 1968; Wilson \& Devinney 1971; Wood 1971; Mochnacki \& Doughty 1972; Rucinski 1973; Hill 1979; Popper \& Etzel 1981; Djurasevic 1992; Drechsel et al. 1994; Hadrava 1997; Bradstreet \& Steelman 2002; Pribulla 2004, Pavlovski et al. 2006, Tamuz et al. 2006). The Wilson \& Devinney code is most often used and is continuously being improved or modified (Kallrath et al.1998; Prša \& Zwitter 2005). The main focus of these codes is to deal with the stars, to determine their properties and their orbit. However, it is often the case that stellar objects are embedded in some moving optically thin environment and/or are accompanied by discs, streams, jets or shells which give rise to various emission spectra. We address these objects with Shellspec.

The SHELLSPEC code is described in more detail in Budaj \& Richards (2004). There has been a lot of progress since that manual was published and an updated version of the manual, with examples of input, output and test runs is available within each new release. A convenient overview may also be found in Budaj \& Richards (2010). 
Major changes since that time include a new model of the reflection effect applicable to tidally distorted and strongly irradiated cold objects (Budaj 2011a), and dust and angle dependent Mie scattering (Budaj 2011b). It includes extinction due to the absorption and scattering as well as the thermal and scattering emission. The original SHELLSPEC code was written in Fortran77 and does not solve the inverse problem of finding the best fit parameters. There are versions of this code in Fortran90 which solve some particular restricted inverse problems (see Tkachenko et al. 2010 and Šejnová et al. 2011). Chadima et al. (2011a) modeled $H \alpha$ emission $\mathrm{V} / \mathrm{R}$ variations caused by discontinuous mass transfer in interacting binaries. Ghoreyshi et al. (2010, 2011) applied the code to the eclipsing binary AV Del. Miller et al. (2007) modeled UV and optical spectra of TT Hya, an Algoltype binary. The sections below describe applications of SHELLSPEC to $\epsilon$ Aur and extrasolar planets. Output from the codes SYNSPEC and COOL-TLUSTY (Hubeny 1988; Hubeny, Lanz \& Jeffery 1994; Hubeny, Burrows \& Sudarsky 2003) is used as a default input of spectra of nontransparent objects, which serves as a boundary condition for the radiative transfer in the interstellar medium.

\section{2. $\epsilon$ Aurigae}

$\epsilon$ Aur is an eclipsing binary with the longest known orbital period, 27.1 yr. A very rare eclipse that lasted for two years is over. However, the object, its origin and, in particular, the secondary component of this binary star remain mysterious. Huang (1965) proposed that the secondary is a dark disk seen edge-on. Wilson (1971) and Carroll et al. (1991) argued that the observed sharp mid-eclipse brightening (MEB) $\dagger$ can only be explained by a tilted disk with a central opening. Ferluga (1990) suggested that the disk is a system of rings.

There has been a wealth of studies during the current eclipse. Orbital solutions were recently revisited by Stefanik et al. (2010) and Chadima et al. (2010). Kloppenborg et al. (2010, 2011) confirmed the dark disk with interferometric observations but they did not confirm the hole in the disk. The spectral energy distribution was studied by Hoard et al. (2010). These authors favor the post AGB+B5V model. Wolk et al. (2010) analyzed Xray observations. Sadakane et al. (2010) carried out the abundance analysis. Recently, Chadima et al. (2010, 2011b) questioned the presence of sharp mid-eclipse brightening and suggested that the photometric variability seen during eclipse is intrinsic to the Fstar. Extremely precise observation of the light-curve of $\epsilon$ Aur were obtained with the Solar Mass Ejection Imager by Clover et al. (2011). These data clearly show a shallow mid-eclipse brightening.

Observations that were used for comparison with our calculations were taken from the AAVSO database (Henden 2011). They were obtained by many observers who contributed to the database during the current eclipse of $\epsilon$ Aur. Only the observations in the V-filter were considered here. These observations also indicate the presence of a shallow mideclipse brightening but it is not as sharp and pronounced as some might have anticipated. Ingress is not as steep as egress, which indicates that the disk is not perfectly symmetric, but suffers from some small disturbance. Its leading part might be more extended or disk slightly inclined out of the orbital plane (warped?).

To explain these observations we suggested an alternative model of $\epsilon$ Aur. Our model of $\epsilon$ Aur consists of two geometrically thick flared disks: an internal optically thick disk

$\dagger$ A mid-eclipse brightening in $\epsilon$ Aur is interpreted by some as only a relatively sharp local maximum near the middle of the eclipse. We proposed a slightly more general definition of the MEB or a new term: mid-eclipse excess (MEE). Our MEB or MEE is a convex feature near the middle of an eclipse bed. A common eclipse has a concave eclipse bed. 


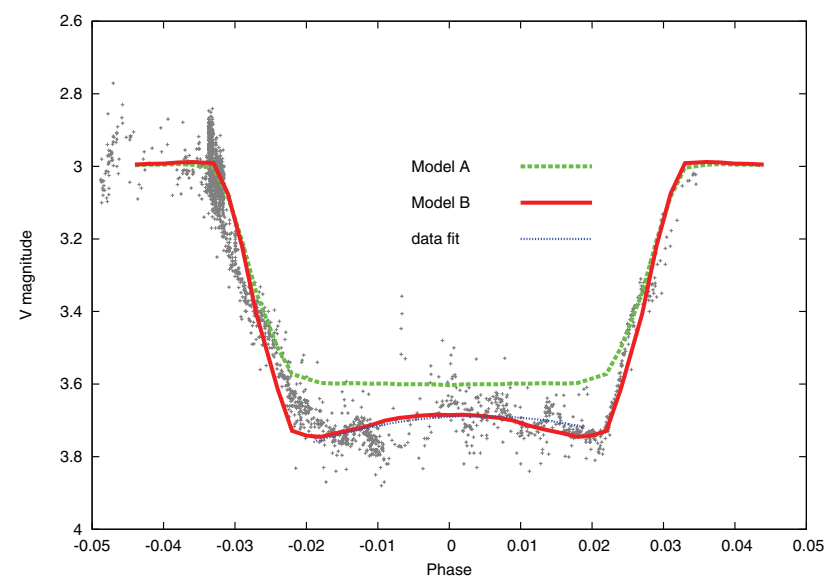

Figure 1. Eclipse of $\epsilon$ Aur by a dark, geometrically thick, flared disk of dust. The disk consists of two parts: (1) The flared optically thick part that causes most of the eclipse, and (2) a flared optically thin part that causes additional absorption, scattering and mid-eclipse brightening. Model (A): Disk has only one part (1). Model (B): disk has both parts part (1) and part (2). Mid-eclipse brightening arises mainly because the edges of the flared disk are more effective in the attenuation of the stellar light than the central parts of the disk. Thin dotted line is a best-fit quadratic function to the eclipse bed. Crosses - observations from AAVSO (Henden 2011).

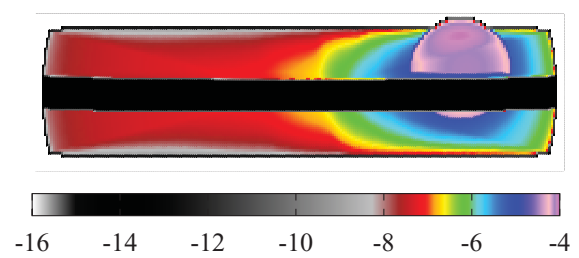

Figure 2. 2D image of $\epsilon$ Aur during eclipse. Black region is a dark, geometrically and optically thick, edge-on flared disk of dust. This disk causes the most of the eclipse. Colored regions correspond to the optically thin flared disk which scatters and absorbs the light from the F-star. Notice that this optically thin disk may produce artificial spots on the surface of the F star.

and an external optically thin disk, which absorbs and scatters radiation. Disks are in the orbital plane and are almost edge-on. The model is based on optical properties of dust grains. It takes into account the extinction due to the Mie scattering and absorption as well as thermal and scattering emission. We argue that there is no need for a highly inclined disk with a hole to explain the current eclipse of $\epsilon$ Aur, even if there is a possible shallow mid-eclipse brightening (see Fig. 1). For more details, kindly see Budaj (2011b). Fig. 2 displays a 2D image of $\epsilon$ Aur as calculated in the $\mathrm{V}$ band.

\section{Shapes of transiting extrasolar planets}

Transiting extrasolar planets are very close to their parent stars. Most of them have circular orbits or very small eccentricity. This indicates that their rotation is synchronous and the classical Roche model can be applied to them. Budaj (2011a) calculated the shape of all transiting exoplanets known at that time. By the shape, we mean the relative proportions of the object. Shape does not change much if the absolute dimensions change within typical measurement errors. Consequently, the new absolute dimensions can be easily linearly rescaled e.g. if the measured cross-section of the planet changes. I use $R_{\text {sub }} / R_{\text {pole }}$ as a measure of the departure from the spherical shape, where $R_{s u b}$ is the 
Table 1. Shapes of the transiting exoplanets. Columns are: $a$-semi-major axis in [AU], $R_{s u b}$ -planet radius at the sub-stellar point, $R_{b a c k}$-planet radius at the anti-stellar point, $R_{\text {pole }}$ -planet radius at the rotation pole, $R_{\text {side }}$-planet radius at the side point, (assumed equal to the planet radius determined from the transit), $R_{e f f}$-effective radius of the planet, $r r=R_{\text {sub }} / R_{\text {pole }}$ -departure from the sphere, $f_{i}=R_{s u b} / L_{1 x}$-fill-in parameter of the Roche lobe, Radii are in units of Jupiter radius. See the text for a more detailed information.

\begin{tabular}{lllllllll}
\hline $\mathrm{a}$ & Rsub & Rback & Rpole & Rside & Reff & rr & $f_{i}$ & name \\
\hline 0.01655 & 1.55474 & 1.54069 & 1.35022 & 1.38600 & 1.42045 & 1.151 & 0.63 & WASP-19 b \\
0.02293 & 1.90438 & 1.89283 & 1.69728 & 1.73600 & 1.77110 & 1.122 & 0.58 & WASP-12 b \\
0.03440 & 2.14496 & 2.14005 & 2.00804 & 2.03700 & 2.06019 & 1.068 & 0.48 & HAT-P-32 b \\
0.02540 & 1.54395 & 1.54167 & 1.47462 & 1.49000 & 1.50166 & 1.047 & 0.42 & CoRoT-1 b \\
0.02312 & 1.40758 & 1.40572 & 1.35013 & 1.36300 & 1.37264 & 1.042 & 0.41 & WASP-4 b \\
0.05150 & 2.04439 & 2.04295 & 1.97515 & 1.99100 & 2.00266 & 1.035 & 0.38 & WASP-17 b \\
0.02250 & 1.23086 & 1.22972 & 1.19084 & 1.20000 & 1.20670 & 1.033 & 0.37 & OGLE-TR-56 b \\
0.03444 & 1.70957 & 1.70825 & 1.65814 & 1.67000 & 1.67862 & 1.031 & 0.36 & WASP-48 b \\
\hline
\end{tabular}

Table 2. Overview of the reflection effect. Scattering refers to the refletion of light off the surface of one of the objects. $A_{b o l}$ is bolometric albedo (Rucinski 1969), $A_{B}$ is Bond albedo, $A_{\nu}$ is monochromatic albedo. MR stands for muliple reflection between surfaces of the two objects.

\begin{tabular}{llll}
\hline Property & Interacting Binaries & Exoplanets & Hybrid model \\
\hline Shape & Roche (limb+grav.dark.) & sphere, rot. ellipsoid & Roche (limb+grav.dark.) \\
Scattering & No & Yes & Yes \\
Albedo & $A_{b o l}$ & $A_{B}, A_{\nu}$ & $A_{B}, A_{\nu}$ \\
(A) Meaning & absorbed=>heating & reflected=>no heating & reflected=>no heating \\
(1-A) Meaning & penetrates into star & heating+heat redistrib. heating+heat redistrib. \\
MR & Yes & No & No \\
\hline
\end{tabular}

radius at the sub-stellar point and $R_{\text {pole }}$ is the radius at the rotational pole. I updated these calculations and recalculated the shape of all 138 transiting exoplanets known as of end of June, 2011. Most of the transiting exoplanets have small departures from a sphere on the order of $1 \%$. However, a fraction of them have departures more than $3 \%$ and these are listed in Table 1. HAT-P-32 b, WASP-12 b, and WASP-19 b are exceptions with departures of about 7,12 , and $15 \%$ respectively. This means that we are observing only a cross section of these planets during the transit. This cross-section is also not spherical, it is characterized by the $R_{\text {side }} / R_{\text {pole }}$ parameter but this is not as high as the $R_{\text {sub }} / R_{\text {pole }}$. Table 1 lists also the effective radius of the planet (radius of the sphere with the same volume) which one could use e.g. for comparison with the theoretical radius calculations. Table 1 illustrates that spherical shape of close-in exoplanets cannot be taken for granted. Moreover, the table demonstrates that although the cross section of the planet might be approximated by an ellipse, the overall Roche shape cannot be approximated by a rotational ellipsoid.

\section{Reflection effect}

The reflection effect operates in very different environments and there are different approaches to model this effect. Table 2 presents an overview of how the models work in the field of interacting binaries and exoplanets. One can see that the models of the reflection effect and definition of the fundamental quantities are very different in these fields. The question is: Is there any transition between the two approaches? What is the 
amount of the heat redistribution? What fraction of light gets reflected off the surface and does not produce heat? Which approach (model) should one choose for a tidally distorted and strongly irradiated cold object? To address these questions and these objects Budaj (2011a) proposed a sort of hybrid model of the reflection effect whose properties are also listed in the table.

\section{Acknowledgements}

JB thanks the VEGA grants No.s 2/0074/9, 2/007810, 2/0094/11.

\section{References}

Bradstreet, D. H. \& Steelman, D. P. 2002, BAAS, 34, 1224

Budaj, J. 2011a, $A J, 141,59$

Budaj, J. 2011b, $A \mathscr{E} A$, 532, L12

Budaj, J. \& Richards, M. T. 2004, Contrib. Astron. Obs. Skalnaté Pleso, 34, 167

Budaj, J. \& Richards, M. T. 2010, ASP-CS, 435, 63

Carroll, S. M., Guinan, E. F., McCook, G. P., \& Donahue, R. A. 1991, ApJ, 367, 278

Chadima, P., Harmanec, P., Yang, S. et al., 2010, IBVS, 5937

Chadima, P., Firt, R., Harmanec, P. et al., 2011a, AJ, 142, 7

Chadima, P., Harmanec, P., Bennett, P. D. et al., 2011b, A\&4A, 530, A146

Clover, J., Jackson, B. V., Buffington, A., Hick, P. P., Kloppenborg, B., \& Stencel, R. 2011, AAS Meeting 217, 257.02

Djurasevic, G., 1992, ApESSS, 197, 17

Drechsel, H., Haas, S., Lorenz, R., \& Mayer, P. 1994, A\&A, 284, 853

Ferluga, S. 1990, A\&A, 238, 270

Ghoreyshi, S. M. R.., Ghanbari, J., \& Salehi, F. 2010, Pub. Astron. Soc. of Australia, 28, 38

Ghoreyshi, S. M. R.., Ghanbari, J., \& Salehi, F. 2011, arXiv:1108.3646

Hadrava, P. 1997, A\& $A S, 122,581$

Henden, A. A., 2011, Observations from the AAVSO International Database, private communication.

Hill, G. 1979, Publ. Dom. Ap. Obs. Victoria, 15, 297

Hoard, D. W., Howell, S. B., \& Stencel, R. E. 2010, ApJ, 714, 549

Hubeny, I. 1988, Computer Physics Comm., 52, 103

Hubeny, I., Burrows, A., \& Sudarsky, D. 2003, ApJ, 594, 1011

Hubeny, I., Lanz, T., \& Jeffery, C. S.: 1994, in Newsletter on Analysis of Astronomical spectra No.20, ed. C.S. Jeffery (CCP7; St. Andrews: St. Andrews Univ.), 30

Huang, S. 1965, ApJ, 141, 976

Kallrath, J., Milone, E. F., Terrell, D., \& Young, A. T. 1998, ApJ, 508, 308

Kloppenborg, B., Stencel, R., Monnier, J. D. et al., 2010, Nature, 464, 870

Kloppenborg, B. K., Stencel, R., Monnier, J. D. et al., 2011, AAS Meeting 217, 257.03

Lucy, L. B. 1968, ApJ, 153, 877

Miller, B., Budaj, J., Richards, M., Koubský, P., \& Peters, G. 2007, ApJ, 656, 1075

Mochnacki, S. W. \& Doughty, N. A., 1972, MNRAS, 156, 51

Pavlovski, K., Burki, G., \& Mimica, P. 2006, A\& A, 454, 855

Popper D. M., Etzel P. B. 1981, AJ, 86, 102

Pribulla, T. 2004, Spectroscopically and Spatially Resolving the Components of Close Binary Stars, R. W. Hidlitch, H. Hensberge and K. Pavlovski, ASP-CS, 318, 117

Prša, A. \& Zwitter, T. 2005, ApJ, 628, 426

Rucinski, S. M. 1969, AcA, 19, 245

Rucinski, S. M. 1973, AcA, 23, 79

Sadakane, K., Kambe, E., Sato, B., Honda, S., \& Hashimoto, O. 2010, PASJ, 62, 1381

Stefanik, R. P., Torres, G., Lovegrove, J. et al., 2010, AJ, 139, 1254

Šejnová, K., Votruba, V., \& Koubský, P. 2011, these proceedings 
Tamuz O., Mazeh T., North P. 2006, MNRAS, 367, 1521

Tkachenko, A., Lehmann, H., \& Mkrtichian, D. 2010, AJ, 139, 1327

Wilson, R. E. 1971, ApJ, 170, 529

Wilson, R. E. \& Devinney, E. J. 1971, ApJ, 166, 605

Wolk, S. J., Pillitteri, I., Guinan, E., \& Stencel, R. 2010, AJ, 140, 595

Wood, D. B. 1971, AJ, 76, 701

\section{Discussion}

R. Wilson: Did you look into the possible importance of multiple scattering? The scattering geometry affects the angular distribution of scattered light and, to a small extent, even whether a scattered photon escapes the atmosphere. The effect is small but might not be negligible.

J. BuDAJ: There is a scattering (reflection) process in two environments. One is the scattering in the atmosphere of stars or nontransparent objects. I do not solve the radiative transfer in the atmospheres of these objects. I take the flux from the model atmosphere calculation and use it to calculate the boundary condition for the radiative transfer in the moving interstellar matter. Models of the atmosphere take the multiple scattering into account. The other is the scattering process in the interstellar medium. In this case, I assume that the medium is optically thin and that it is irradiated by one or two sources. I take into account only the first scattering event. In the optically thin medium, the probability of the second and higher scattering events rapidly decreases. To take them into account, one would have to solve the $3 \mathrm{D}$ radiative transfer in the $3 \mathrm{D}$ moving medium.

A. Burrows: Have you thought of using your code to calculate light curves for WASP$12 \mathrm{~b}$ at the warm Spitzer bands $(3.6 \mathrm{~m}$ and $4.5 \mathrm{~m})$, instead of at $8 \mathrm{~m}$ ? There should be some data soon from Spitzer for WASP-12b at those bands and such models would be relevant.

J. Budaj: No. However, this would be indeed very interesting. Thank you for the suggestion/information.

S. RUCINSKI: I congratulate you for reorganizing the definitions of albedo as applied to stars and planets. The old definition was directly related to the so-called "reflection effect" in eclipsing binaries.

\section{J. BudAJ: Thank you.}

J. Southworth: You find that the difference in radius between a spherical planet and the substellar point of a Roche-model planet is as large as $15 \%$. When a planet is transiting, we do not see its substellar point but we do measure its equatorial/ polar radius. This will be closer to the radius of a spherical planet so the problem is not as bad. Can you put a figure on this?

J. BudAJ: Yes. That is true (if one deals with transits and not with other phases). $R_{\text {side }} / R_{\text {pole }}$ is not as big. I do not remember the exact numbers but they are in my paper for all the transiting planets. This ratio might be about $3 \%$ in the worst case. The effective radius of the planet (radius of the sphere with the same volume) is also tabulated there. 